\title{
Vigilancia de brotes de enfermedades transmitidas por alimentos en Chile
}

\author{
Andrea Olea, Janepsy Díaz, Rodrigo Fuentes, Alejandra Vaquero y Maritza García
}

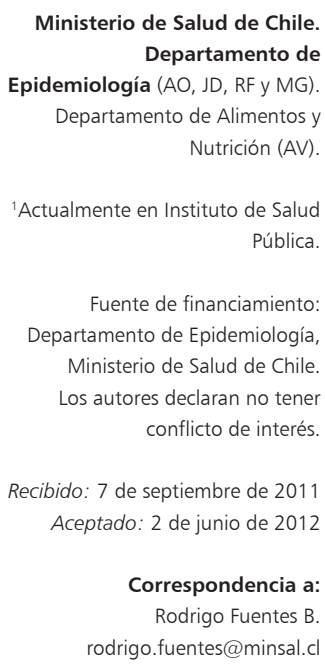

Ministerio de Salud de Chile. Departamento de Epidemiología (AO, JD, RF y MG). Departamento de Alimentos y Nutrición (AV)

'Actualmente en Instituto de Salud Pública.

Fuente de financiamiento: Departamento de Epidemiología, Ministerio de Salud de Chile. Los autores declaran no tener conflicto de interés.

Recibido: 7 de septiembre de 2011 Aceptado: 2 de junio de 2012

Correspondencia a: Rodrigo Fuentes $B$. rodrigo.fuentes@minsal.cl

\section{Foodborne disease outbreaks surveillance in Chile}

Background: Foodborne disease outbreaks are one of the main health problems globally, having an extensive impact on human welfare. The World Health Organization considers them as the main cause of morbidity and mortality in developing countries, and responsible for high levels of loss of productivity in developed countries. Aim: To describe the epidemiology of foodborne disease outbreaks according to data contained in an automated surveillance system. Method: Descriptive observational study of notified outbreaks from the surveillance system, between 2005 and 2010 in Chile. The information was based on etiology, temporal and spatial distribution, and epidemiologic description of outbreaks during this period. Results: There were 5,689 notified outbreaks. Most of them occurred during 2006 (1,106 outbreaks, rate 6.7 per 100,000 inhabitants) and 2008 (1,316 outbreaks, rate 7.9 per 100, 000 inhabitants) with an increase during summer. Fifty four percent occurred in the Metropolitan region. The group aged 15 to 44 years old, was the most affected one. Sixty four percent of the outbreaks had the food involved registered, of which fish and fishery products reached $42 \%$. An $81 \%$ of the outbreaks did not have a precise etiologic diagnosis. Of all patients involved, 97\% were outpatients, 3,2\% were hospitalized patients, and $0,1 \%$ died. Only $49 \%$ of the outbreaks had information about the lack of food safety, with a $34,1 \%$ related to food handling procedures. Conclusions: Through the information on the epidemiology of foodborne diseases obtained by the Chilean surveillance system, appropriate control measures could be taken.

Key words: Health surveillance system, infectious disease outbreak, foodborne diseases, food handling, food contamination, salmonella, vibrio, Chile.

Palabras clave: Sistema de vigilancia sanitaria, enfermedades transmitidas por alimentos, manipulación de alimentos, contaminación de alimentos, Salmonella spp, Vibrio spp, Chile.

\section{Introducción}

e considera a las enfermedades transmitidas por alimentos (ETA), como una importante carga de enfermedad en el mundo. La Organización Mundial de la Salud (OMS) señala que en países menos desarrollados, las ETA son la principal causa de enfermedad y muerte, asociadas a una carga socio-económica significativa $^{1,2}$. En los países desarrollados, las ETA son responsables de altos niveles de pérdida de productividad, costos asociados al uso de los servicios de salud y a la implementación y monitoreo de políticas de inocuidad de los alimentos ${ }^{3}$.

Aproximadamente $70 \%$ de las diarreas se originan por la ingestión de alimentos contaminados con microorganismos o toxinas. Se ha descrito alrededor de 250 agentes causantes de ETA, entre los que se incluyen bacterias, virus, hongos, parásitos, priones, toxinas y metales ${ }^{4}$. Los cambios en los hábitos alimentarios de la sociedad, como el consumo de alimentos envasados, comidas fuera del hogar, expendio de comidas preparadas y comidas rápidas, son factores que contribuyeron al incremento de las ETA.
Estos cambios en los estilos de vida revisten riesgos, fundamentalmente en las sociedades desarrolladas. En comunidades con menor nivel socio-económico siguen siendo prevalentes las enfermedades entéricas como el cólera, la fiebre tifoidea y los parásitos ${ }^{5}$. La posible relación entre factores socio-demográficos como el envejecimiento de la población también podría estar influyendo en la aparición de estas enfermedades ${ }^{6,7}$.

La OMS estima que en el mundo, la incidencia anual de diarreas es de 1.500 millones de casos, y 3 millones de niños bajo 5 años de edad mueren anualmente ${ }^{1}$. En Estados Unidos de América (E.U.A.), se estima que alrededor de 76 millones de personas se enferman anualmente de una ETA, 325.000 se hospitalizan y 5.000 mueren, implicando costos significativos dentro de los gastos en salud ${ }^{8}$. Por otro lado, el Centro para el Control y la Prevención de Enfermedades (CDC) de Atlanta, Georgia, analizó la información contenida en 1.100 brotes de ETA ocurridos durante el año 2007 en E.U.A. Estos brotes causaron más de 21.000 enfermos y 18 muertes $^{9}$. Sin embargo, se desconoce la real magnitud de los brotes de ETA, dado que son pocas las personas que consultan a centros asistenciales 
por síntomas gastrointestinales (usualmente los reportes oficiales sólo representan a este pequeño porcentaje). Por otra parte, sólo a una fracción de estos consultantes se les extrae muestras clínicas para la determinación del agente causal. A pesar de lo anterior, la "pirámide de carga de enfermedad" se puede determinar mediante los datos de atención médica, toma de muestra, análisis de laboratorio, confirmación de laboratorio y reporte al sistema oficial de vigilancia ${ }^{10,11}$. En Chile, Región Metropolitana (2008), se llevó a cabo un estudio de carga de enfermedad gastrointestinal aguda que mostró una prevalencia mensual ajustada por edad de 9,2\%, similar a lo observado en estudios en Canadá y Cuba, y ligeramente superior a lo observado en estudios en E.U.A., Australia y Argentina ${ }^{12}$.

La vigilancia y control de las ETA es considerado por la OMS un componente importante en el PLAN DE ACCIÓN dentro del marco del Reglamentario Sanitario Internacional (RSI). El enfoque básico está orientado a la asistencia técnica y económica a los países necesitados para el establecimiento de un Sistema de Vigilancia Epidemiológica de ETA que permita detectar oportunamente la aparición de brotes, conocer la magnitud del problema, diseñar estrategias de prevención y control de las mismas, y determinar la posibilidad de afectar a otros Estados Miembros ${ }^{13}$. En Chile, de acuerdo al Reglamento sobre Notificación de Enfermedades Transmisibles de Declaración Obligatoria*, los brotes de ETA son de notificación obligatoria, con el propósito de constituir un sistema de información que apoye las acciones de salud pública en la prevención y control de estas enfermedades ${ }^{14}$. En el año 2005, entró en vigencia el actual sistema de vigilancia de las ETA en Chile, con el propósito de disponer de un sistema automatizado que permitiera el fácil acceso y uso para la notificación de estos brotes.

El objetivo de este artículo es la descripción del comportamiento epidemiológico de las ETA en Chile, a partir de los resultados que entrega el sistema automatizado de vigilancia de ETA desarrollado por los Departamentos de Epidemiología y de Alimentos y Nutrición del Ministerio de Salud de Chile.

\section{Materiales y Método}

Estudio observacional de tipo descriptivo, inserto en una investigación operacional dentro del sistema de vigilancia de las ETA. El objeto de estudio se conformó por todos los brotes de ETA notificados en Chile a través del sistema automatizado de vigilancia de ETA dependiente del Departamento de Estadísticas e Información de Salud (DEIS) del Ministerio de Salud (MINSAL), entre los años 2005 y 2010. La descripción de la información se basó en el aspecto etiológico (agente causal, alimento involucrado, tipo de local de consumo, pérdida de la inocuidad), la distribución temporal (estacionalidad anual), distribución espacial (por región) y descripción epidemiológica (en base a la edad, sexo y sintomatología más frecuente de los casos, además de la gravedad) de los brotes notificados durante dicho período.

La base de datos con los registros de brotes investigados obtenida desde el DEIS fue analizada utilizando el paquete Excel y el programa Epinfo 2004 (Versión 3.2). La información resultante del análisis fue expresada en frecuencias absolutas, porcentajes, proporciones o tasas, y representada en tablas y gráficos para su mejor comprensión. Para analizar la estacionalidad, se consideró como "alza en el número de brotes" si al menos en dos meses consecutivos el número fuera mayor que los meses precedentes.

\section{Resultados}

Entre los años 2005 y 2010 se notificaron en el sistema de vigilancia automatizado 5.689 brotes de ETA. Los años 2006 y 2008 registraron el mayor número de brotes, contabilizándose 1.106 (6,7 brotes por 100.000 hab) y 1.316 (7,9 brotes por 100.000 hab) brotes, respectivamente. Posteriormente, se observó una disminución en los años 2009 y 2010, siendo notificados 910 (5,4 brotes por 100.000 hab) y 741 (4,3 brotes por 100.000 hab) brotes de ETA, respectivamente (Tabla 1). Se observó además el comportamiento estacional habitual de la enfermedad en el quinquenio en estudio, con aumento de brotes en los meses de verano (enero y febrero), período que concentró entre 42 y $50 \%$ del total de brotes del año.

E1 78\% de los brotes nacionales registró algún diagnóstico de la Clasificación Internacional de Enfermedades, décima versión (CIE-10) (Figura 1). De esta proporción, $65 \%$ correspondió a "ETA bacteriana no especificada" (CIE 10: A05.9) mientras que $10 \%$ fue registrada como "Diarrea y gastroenteritis de presunto origen infeccioso" (DGPOI) (CIE 10: A09), es decir, 81\% del total de brotes analizados no tuvo un diagnóstico etiológico preciso. Dentro de las etiologías más relevantes se destacan "Intoxicación alimentaria debido a Vibrio parahaemolyticus" (CIE 10: A05.3) y a "Salmonella Enteritidis" (CIE 10: A02.0 y A02.9), con 9 y 3\%, respectivamente.

Respecto a los brotes causados por " $V$. parahaemolyticus", $31 \%$ de ellos ocurrió el año 2006 y 42\% en el año 2007, para luego disminuir su incidencia y alcanzar sólo $2 \%$ el año 2010. Por el contrario, se observó un aumento de brotes por "S. Enteritidis", de 8\% el año 2005 a 34\% en el año 2010.

\footnotetext{
* Decreto Supremo No 158 / 2004 del Ministerio de Salud, publicado en el Diario Oficial el 10 de mayo de 2005.
} 


\begin{tabular}{|c|c|c|c|c|c|c|c|c|c|c|c|c|}
\hline \multirow[t]{2}{*}{ Región } & \multicolumn{2}{|c|}{ Año 2010} & \multicolumn{2}{|c|}{ Año 2009} & \multicolumn{2}{|c|}{ Año 2008} & \multicolumn{2}{|c|}{ Año 2007} & \multicolumn{2}{|c|}{ Año 2006} & \multicolumn{2}{|c|}{ Año 2005} \\
\hline & $\begin{array}{l}\mathrm{N}^{\circ} \text { de } \\
\text { brotes }\end{array}$ & Tasa* & $\begin{array}{l}\mathrm{N}^{\circ} \text { de } \\
\text { brotes }\end{array}$ & Tasa* & $\begin{array}{l}\mathrm{N}^{\circ} \text { de } \\
\text { brotes }\end{array}$ & Tasa* & $\begin{array}{l}\mathrm{N}^{\circ} \text { de } \\
\text { brotes }\end{array}$ & Tasa* & $\begin{array}{l}\mathrm{N}^{\circ} \text { de } \\
\text { brotes }\end{array}$ & Tasa* & $\begin{array}{l}\mathrm{N}^{\circ} \text { de } \\
\text { brotes }\end{array}$ & Tasa* \\
\hline Arica y Parinacota & 31 & 16,8 & 0 & 0,0 & 1 & 0,5 & 4 & 2,1 & 5 & 2,6 & 3 & 1,6 \\
\hline Tarapacá & 21 & 6,7 & 15 & 4,9 & 6 & 2,0 & 2 & 0,7 & 8 & 2,8 & 7 & 2,5 \\
\hline Antofagasta & 5 & 0,9 & 11 & 1,9 & 23 & 4,1 & 21 & 3,8 & 18 & 3,3 & 13 & 2,4 \\
\hline Atacama & 3 & 1,1 & 24 & 8,6 & 48 & 17,4 & 32 & 11,7 & 46 & 16,9 & 13 & 4,8 \\
\hline Coquimbo & 7 & 1,0 & 4 & 0,6 & 16 & 2,3 & 7 & 1,0 & 19 & 2,8 & 32 & 4,8 \\
\hline Valparaíso & 163 & 9,3 & 244 & 14,0 & 276 & 16,0 & 329 & 19,3 & 188 & 11,2 & 20 & 1,2 \\
\hline Metropolitana & 364 & 5,3 & 466 & 6,8 & 696 & 10,3 & 493 & 7,4 & 640 & 9,7 & 407 & 6,2 \\
\hline O’Higgins & 11 & 1,2 & 1 & 0,1 & 20 & 2,3 & 13 & 1,5 & 26 & 3,1 & 6 & 0,7 \\
\hline Maule & 24 & 2,4 & 10 & 1,0 & 44 & 4,4 & 35 & 3,6 & 43 & 4,4 & 12 & 1,2 \\
\hline Bío Bío & 74 & 3,6 & 79 & 3,9 & 113 & 5,6 & 77 & 3,9 & 69 & 3,5 & 45 & 2,3 \\
\hline Araucanía & 6 & 0,6 & 3 & 0,3 & 14 & 1,5 & 9 & 1,0 & 6 & 0,6 & 8 & 0,9 \\
\hline Los Ríos & 25 & 6,6 & 41 & 10,8 & 41 & 10,9 & 6 & 1,6 & 11 & 2,9 & 8 & 2,1 \\
\hline Los Lagos & 0 & 0,0 & 0 & 0,0 & 0 & 0,0 & 1 & 0,1 & 6 & 0,8 & 1 & 0,1 \\
\hline Aysén & 0 & 0,0 & 10 & 9,6 & 14 & 13,6 & 2 & 2,0 & 18 & 17,9 & 1 & 1,0 \\
\hline Magallanes & 7 & 4,4 & 2 & 1,3 & 4 & 2,5 & 4 & 2,5 & 3 & 1,9 & 5 & 3,2 \\
\hline Total País & 741 & 4,3 & 910 & 5,4 & 1.316 & 7,9 & 1.035 & 6,2 & 1.106 & 6,7 & 581 & 3,6 \\
\hline
\end{tabular}

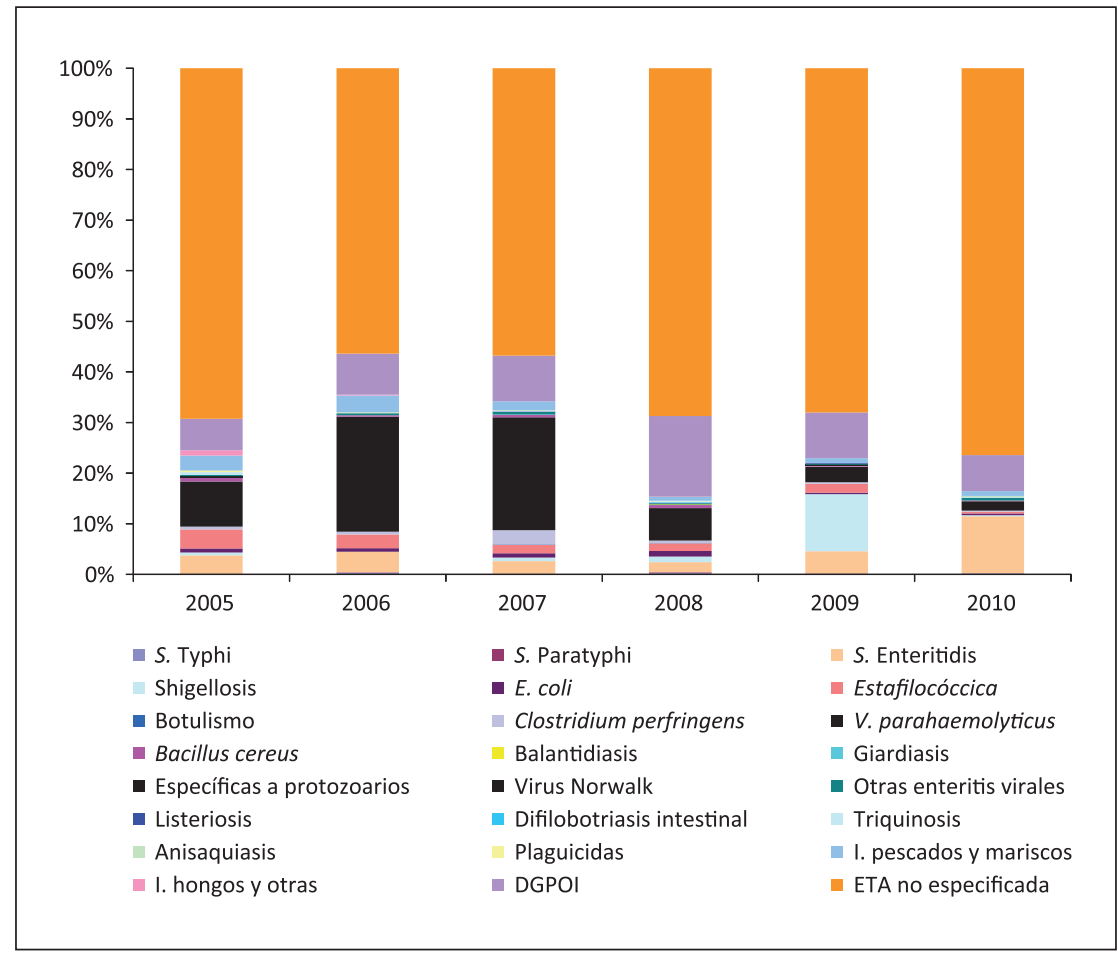

Figura 1. Distribución de brotes de ETA anual según agente etiológico. Chile, 2005-2010.
Al analizar la ocurrencia de brotes por región durante el período 2005-2010, la Región Metropolitana registró el mayor número de brotes de ETA (3.066 brotes) seguido por la Región de Valparaíso (1.220 brotes), lo que representó 54 y $21 \%$ del total de brotes notificados en el país para dicho período, respectivamente. Los agentes etiológicos mayormente involucrados en ambas regiones fueron: V. parahaemolyticus (64\%), Salmonella spp. (11\%), Shigella spp. (6\%) Shigella sonnei (5\%) y otros, como por ejemplo, el único brote por Listeria monocytogenes ocurrido en el año 2008, que afectó a 119 personas, mayoritariamente mujeres embarazadas, ancianos e inmunodeprimidos. El estudio de las cepas de este último brote fue llevado a cabo por el Instituto de Salud Pública (ISP), concluyendo que 55\% de los casos correspondió a una cepa genéticamente relacionada (clon 009), considerada la cepa epidémica causante del brote.

En el análisis de los seis años, las mayores incidencias acumuladas de brotes según región fueron reportadas para las regiones de Atacama, Valparaíso, Metropolitana y Los Ríos. Específicamente durante el año 2010, la incidencia acumulada más alta fue alcanzada por la Región de Arica y Parinacota (16,8 por 100.000 hab) seguida por la Región de Valparaíso (9,3 por 100.000 hab). A pesar de que la Región Metropolitana representó 49\% del total de brotes 
notificados para el año 2010, la incidencia acumulada fue de 5,3 por 100.000 hab. Las regiones de los Lagos y Aysén no notificaron brotes durante ese año (Figura 2).

Durante los años 2008 y 2009 se registró el mayor número de personas afectadas por brotes de ETA (7.949 y 10.151 involucrados, respectivamente), traducido en un promedio de 6 personas por brote el año 2008 y 11 personas por brote en el año 2009. El año 2010 en cambio, registró el menor número de personas enfermas (2.245), lo que significó un promedio de 3 personas por brote. El análisis en base a grupos de edad reportó que en el período analizado, la incidencia acumulada del grupo de edad más afectado correspondió al segmento de 15 a 44 años (rango: 30,8 a 60,6 por 100.000 hab) exceptuando el año 2009, donde el grupo de 5 a 14 años registró el valor más alto $(37,8$ por 100.000 hab). La distribución por sexo fue similar en todo el período (49,7\% mujeres y 50,3\% hombres) y a través de los años.

La sintomatología más frecuentemente reportada correspondió a diarrea y dolor abdominal (ambos en $73 \%$ de los casos), seguido por náuseas o vómitos (68\%) y fiebre (19\%) (Figura 3).

De acuerdo a la gravedad de los brotes notificados, 97\% correspondió a pacientes ambulatorios, 3,2\% fueron hospitalizados y $0,1 \%$ falleció a causa de la ETA. Del total de casos reportados, las tasas de hospitalización anuales más altas según grupo de edad fueron reportadas por el grupo $<1$ año (rango: 0,0 a 43,4 por 100 enfermos bajo 1 año de edad); sin embargo, el grupo de 1 a 4 años registró los valores más altos durante el año 2009 y 2006 (7,6 y 10 por 100 enfermos de 1 a 4 años, respectivamente). Por otro lado, la tasa de letalidad por ETA más alta se registró el año 2008 (0,17 por 100 enfermos) mientras que la tasa más baja se reportó el año $2007(0,03$ por 100

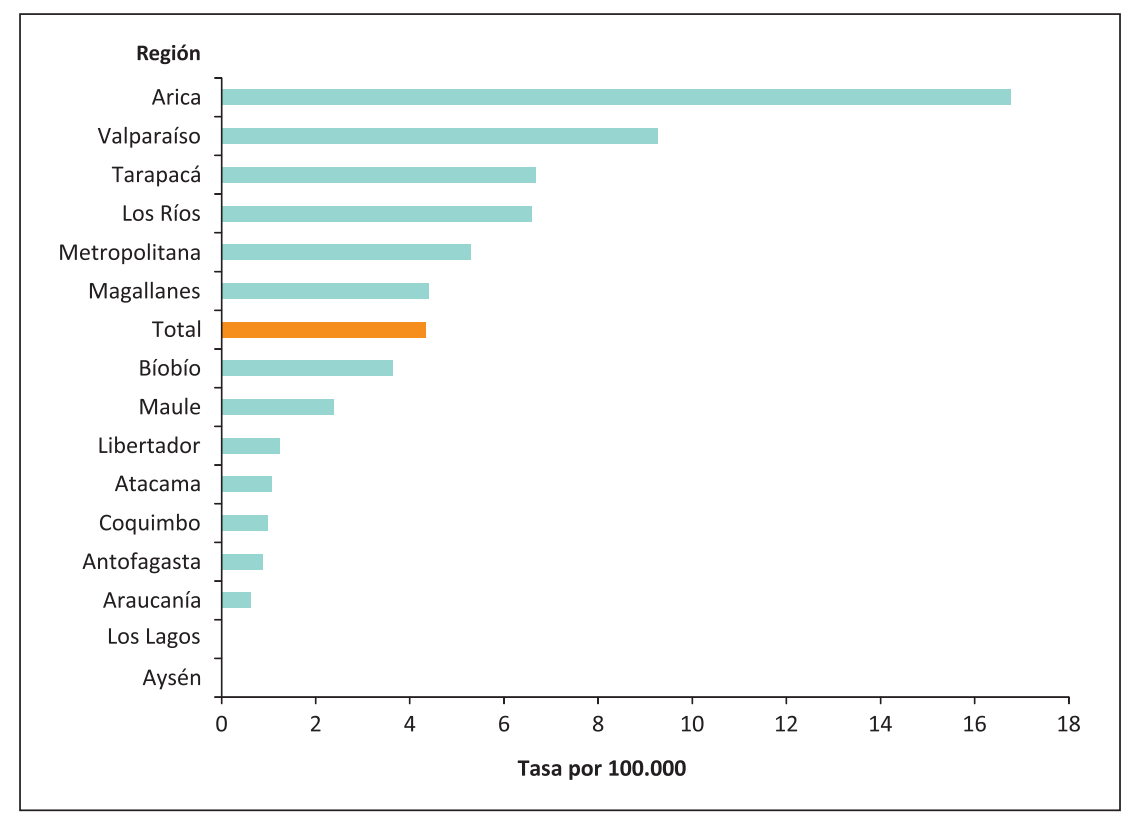

Figura 2. Tasa de notificación acumulada de brotes de ETA según región. Chile, 2010.

enfermos). El análisis por grupos de edad consignó que la tasa de letalidad durante los años 2005, 2007 y 2010 fue mayor en el grupo de 1 a 4 años $(0,63 ; 0,79 ;$ y 0,65 por 100 enfermos, respectivamente), el año 2006 fue para el grupo de 15 a 44 años (0,18 por 100 enfermos), el año 2008 fue para los < de 1 año (4,35 por 100 enfermos) y el año 2009 fue para los de 65 años o más $(1,15$ por 100 enfermos).

El $64 \%$ de los brotes notificados durante el período en estudio reportó información respecto al alimento involucrado. De ellos, los pescados y productos de la pesca

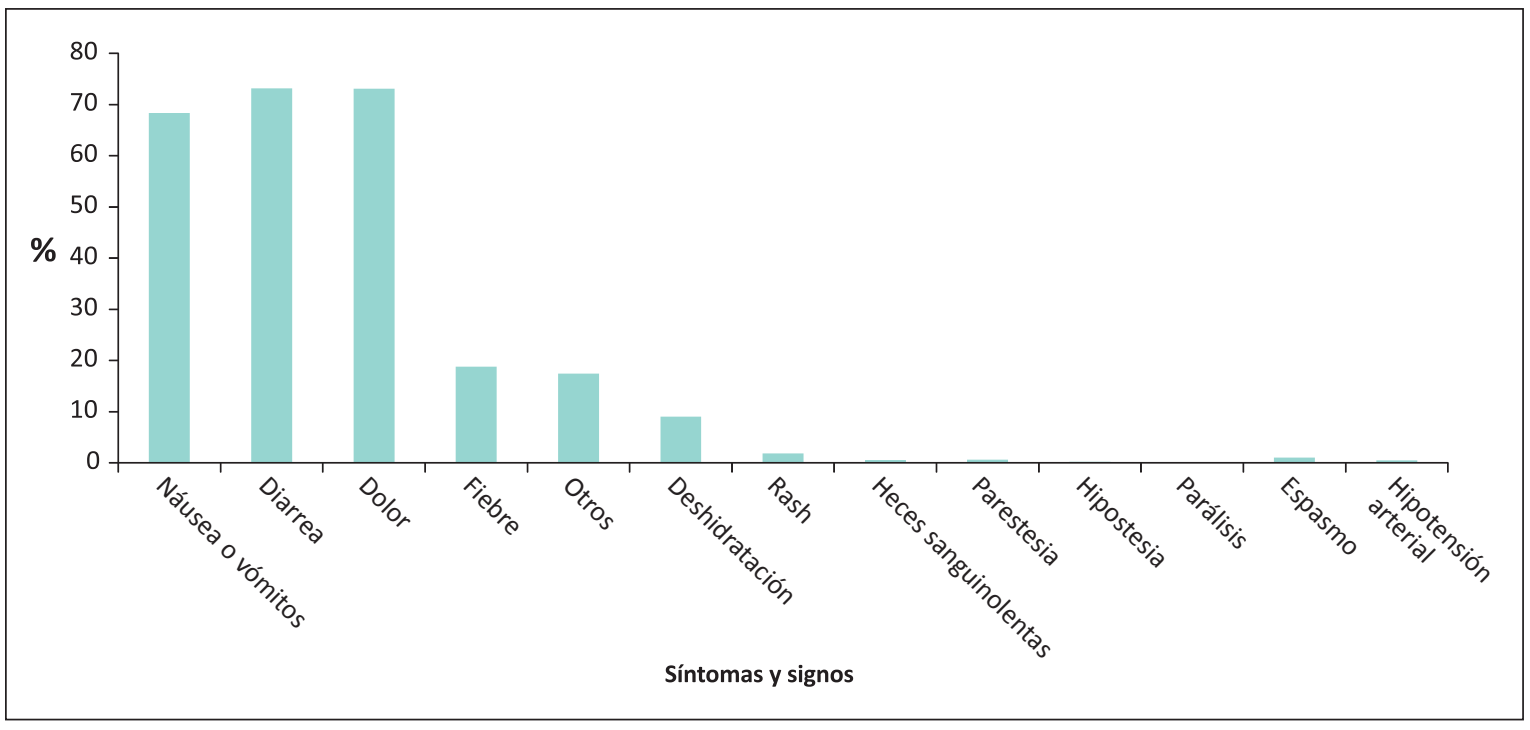

Figura 3. Síntomas y signos más frecuentes reportados en los brotes de ETA. Chile, 2005-2010. 
alcanzaron la mayor proporción (42\%), y dentro de este grupo, $72 \%$ correspondió a moluscos bivalvos crudos. De los brotes con información alimentaria, 21\% registró consumo de comidas y platos preparados; los ocasionados por consumo de carnes o productos cárnicos alcanzaron a $11 \%$, y de estos últimos, las carnes de aves fueron las causantes de $36 \%$ de los brotes.

Respecto a la identificación del local de consumo de alimentos contaminados, esta información estuvo presente en $72 \%$ del total de brotes notificados. El mayor porcentaje lo obtuvo el hogar (29,3\%) seguido de restaurantes $(12,8 \%)$ y casinos $(4,9 \%)$, orden que se mantiene en los análisis por año.

En los cinco años analizados, sólo 49\% de los brotes registró el dato de pérdida de inocuidad del alimento. El proceso de manipulación del alimento fue la causa de $34,1 \%$ y la pérdida de inocuidad durante el proceso de producción dio cuenta de 11,3\%. El año 2005 se registró el valor más alto para el proceso de manipulación comercial (59,2\%); sin embargo, ese mismo año se registró el valor más bajo para el proceso de manipulación doméstica $(13,1 \%)$ y para el proceso de producción $(12,4 \%)$. La tendencia a lo largo de los años indica una disminución en la proporción de la pérdida de inocuidad atribuible a la manipulación comercial pero un aumento en el valor de manipulación doméstica y producción, alcanzando al año 2010 valores de 30,6-26,1 y 18,1\%, respectivamente.

\section{Discusión}

Las ETA, lejos de ser un problema del pasado, se han convertido en un problema emergente. La globalización de los mercados y la complejidad de la cadena alimentaria hacen que la disponibilidad de alimentos seguros constituya cada vez más una ardua tarea en el mundo pleno de patógenos, alergenos o contaminantes ambientales.

La creación de un sistema automatizado de vigilancia de ETA permitió el seguimiento, la recolección sistemática, análisis e interpretación de los datos sobre el evento y sus condiciones relacionadas, incluyendo como elementos básicos la difusión de dicha información a los entes que necesitan conocerla para lograr a través de ella una acción de prevención y control más efectiva y dinámica en los diferentes niveles de control ${ }^{15}$.

Esta vigilancia consistió en un proceso sistémico, ordenado y planificado de observación y medición de ciertas variables definidas ${ }^{16}$, permitiendo demostrar que el sistema desempeña la función para el cual fue creado, proporcionando a quienes toman las decisiones, fundamentar las mismas en evidencias científicas y destinar los recursos según las prioridades sanitarias del país ${ }^{17}$.

En este estudio se identificó a $V$. parahaemolyticus como el agente causal de mayor importancia en los años
2006 y 2007, mientras que en los años 2009 y 2010 aumenta el porcentaje de brotes asociado a $S$. Enteritidis. Este comportamiento es similar al descrito por países europeos, donde Salmonella spp. ocupa el primer lugar entre los agentes causales de ETA ${ }^{18}$. Diferente comportamiento se observa en E.U.A., donde norovirus ocupa el primer lugar, seguido por Salmonella $\mathrm{spp}^{19}$.

Sin embargo, en Chile, no es posible dimensionar la prevalencia de brotes de ETA asociado a agentes virales debido a que no se realizan estudios de laboratorio para determinar este tipo de agentes en los brotes sospechosos. Se considera además, como otro impedimento, la escasez en el muestreo de alimentos y sus limitantes en el estudio viral de las muestras ambientales. Por ejemplo, en la Región Metropolitana, sólo en $2 \%$ de los brotes investigados se logró identificar a agentes virales como la causa del brote; a nivel país, los brotes diagnosticados como "Otras enteritis virales" fueron sólo $0,3 \%$, sin determinación de su agente causal.

No se puede dejar de mencionar la ocurrencia de un brote de gastroenteritis aguda asociado a calicivirus, ocurrido en la Región de Antofagasta (entre el 8 de marzo y 28 de abril de 2010). Se trató de un brote de gran magnitud, que generó $31.036 \operatorname{casos}^{20}$. En este brote fue crucial la toma de muestras, tanto clínicas como ambientales, para así determinar el agente y la fuente de contaminación, lo que además permitió aplicar las medidas correctoras para cortar la cadena de transmisión y evitar así la aparición de más casos. Este brote no fue notificado en el sistema de vigilancia de ETA por parte del equipo regional, debido a que se interpretó como un brote de contaminación ambiental.

Los resultados obtenidos del análisis de los brotes de ETA notificados en el sistema nos permiten concluir que no todos los casos que presentan gastroenteritis aguda asociada a una ETA acuden a un centro de salud a recibir atención médica. Este hecho dificulta el conocimiento de la real magnitud de los brotes ocurridos. Estudios realizados en la Región Metropolitana de Chile (año 2008) muestran que sólo $22 \%$ de los afectados por gastroenteritis acudió a un centro de atención médica ${ }^{12}$. La Encuesta de Calidad de Vida y Salud 2008, reportó que 24\% de los encuestados presentó enfermedad o molestia digestiva en las últimas dos semanas previas a la encuesta sin haber acudido a recibir atención en establecimientos de salud $^{21}$. Esta podría ser la causa que esté influyendo en los resultados, los que reportaron que el número de afectados por brote va en un rango de 3 a 7 enfermos.

Los brotes estudiados reflejaron que el mayor porcentaje se asoció a la inadecuada manipulación de alimentos durante su comercialización o preparación doméstica, lo cual está relacionado a la deficiente conservación de los alimentos y hábitos de consumo. Se considera que en la población chilena existe una baja cultura sanitaria 
en los manipuladores de alimentos, incluyendo tanto el nivel comercial como domiciliario. Esto no difiere de lo reportado por la literatura científica, donde se plantea un incremento importante de estas deficiencias en los últimos años, poniendo de manifiesto la necesidad de mejorar las políticas alimentarias desde la preparación hasta la prohibición de algunos alimentos en establecimientos comerciales ${ }^{22}$. Los autores consideramos que promover las actividades de educación sanitaria sería la estrategia más eficiente para evitar estas deficiencias, aunque se considera una medida de impacto a mediano y largo plazo. Otro elemento de importancia para evitar la ocurrencia de estos brotes lo constituye la mantención de los alimentos en la cadena de frío hasta su consumo domiciliario. La educación del consumidor es necesaria, no sólo para mantener la cadena de frío, sino también para lograr una cocción adecuada de los alimentos y para la adopción de medidas tendientes a evitar la contaminación cruzada. Todas las partes involucradas en la cadena de producción, desde el productor al consumidor, comparten la responsabilidad de la inocuidad del alimento, según lo plantea el Codex Alimentarius de la $\mathrm{FAO}^{23}$.

Los hallazgos relacionados al alimento involucrado (pescados y productos de la pesca, y las carnes o productos cárnicos) no difieren mayormente con los brotes de ETA reportados por el CDC en E.U.A., donde los alimentos involucrados de mayor relevancia fueron: pescados, moluscos y carnes ${ }^{24}$. No obstante, se debe mejorar tanto la identificación del alimento involucrado en los brotes de ETA como del agente infectante en el alimento, lo cual sería posible a través de una intervención e investigación oportuna $^{25}$. En base a lo anterior, el sistema de vigilancia de ETA indicó que en los dos últimos años (2009 y 2010), las mayores prevalencias en el tipo de alimento involucrado (cuando fue reportado) estuvo asociada al consumo de moluscos $(24 \%)$, comidas preparadas $(13 \%)$, carne de ave $(9 \%)$ y mayonesa elaborada a partir de huevos frescos (casera) (10\%). Nuestros resultados son similares a lo encontrado en referencias revisadas; por ejemplo, el Sistema de Vigilancia de Enfermedades Transmitidas por Alimentos que mantiene el CDC en sus informes, ha implicado a las comidas preparadas que contienen huevos como el alimento responsable de estos brotes ${ }^{9}$.

Las ETA, de acuerdo a su comportamiento epidemiológico actual, seguirán requiriendo de nuestro esfuerzo y creatividad para lograr actuar oportunamente y proponer medidas de control acordes con la globalización de los productos alimenticios y la re-emergencia de patógenos. El sistema de vigilancia ha permitido conocer el comportamiento epidemiológico de las ETA en Chile, identificar los grupos vulnerables, la magnitud del problema y construir la pirámide de carga de enfermedad, permitiendo la adop- ción de medidas de control y prevención correspondientes.

Dentro de las medidas que los autores recomiendan se encuentran: reforzar la vigilancia estimulando la investigación y notificación de los brotes de ETA con el objeto de mejorar la calidad de la información; aumentar las labores de fiscalización dirigidas a la cadena de elaboración, distribución y expendio de los alimentos, por parte de las Autoridades Sanitarias regionales; desarrollar acciones de prevención y coordinación intersectorial dirigidas a los consumidores y elaboradores de alimentos; desarrollar campañas comunicacionales orientadas al lavado de manos, evitar la contaminación cruzada y consumir productos de riesgo cocidos, entre otros, de tal forma de actuar sobre los factores contribuyentes que facilitan el proceso de infección y así adoptar las medidas necesarias para garantizar la inocuidad de los alimentos. Del mismo modo, consideramos importante realizar estudios de carga de enfermedad atribuible a las ETA y desarrollar metodologías analíticas que permitan el desarrollo de estudios virales tanto clínicos como ambientales.

\section{Resumen}

Antecedentes: Las enfermedades transmitidas por alimentos (ETA) son una importante carga de enfermedad en el mundo. La OMS las señala como la principal causa de enfermedad y muerte en países en desarrollo, mientras que en países desarrollados son responsables de altos niveles de pérdida de productividad. Objetivo: Describir epidemiológicamente los brotes de ETA chilenos de acuerdo a la información contenida en un sistema automatizado de vigilancia. Método: Estudio observacional descriptivo de los brotes notificados en el sistema de vigilancia, entre los años 2005 y 2010 en Chile. La descripción se basó en el aspecto etiológico, distribución temporal y espacial, y descripción epidemiológica de los brotes durante dicho período. Resultados: Se notificaron 5.689 brotes. La mayoría se presentó durante el 2006 (1.106 brotes, tasa $6,7$ por $100.000 \mathrm{hab})$ y 2008 (1.316 brotes, tasa 7,9 por $100.000 \mathrm{hab}$ ) con un aumento en los meses de verano. El $54 \%$ ocurrió en la Región Metropolitana. El grupo de 15 a 44 años fue el más afectado. Del 64\% que registró el alimento involucrado, pescados y productos de la pesca alcanzaron el $42 \%$. Un $81 \%$ del total de brotes no tuvo un diagnóstico etiológico preciso. Del total de pacientes, $97 \%$ fueron ambulatorios, $3,2 \%$ se hospitalizaron, y $0,1 \%$ fallecieron. Sólo $49 \%$ de los brotes registró pérdida de inocuidad del alimento, siendo el mayor porcentaje (34,1\%) atribuible al proceso de manipulación del alimento. Conclusiones: El sistema de vigilancia chileno permitió conocer el comportamiento epidemiológico de las ETA, y facilitó la adopción de medidas de control oportunas. 


\section{Referencias bibliográficas}

1.- WHO. 2007. Consultation to develop a strategy to estimate the global burden of foodborne diseases. World Health Organization, Geneva, Switzerland. Available at:http://www.who.int/ foodsafety/publications/foodborne_disease/ burden_sept06/en/index.html (Consultado: 20/04/2011).

2.- Hall G V, Kirk M D, Ashbolt R, Stafford R, Lalor K. Frequency of infectious gastrointestinal illness in Australia, 2002: regional, seasonal and demographic variation. Epidemiol Infect 2006; 134 (1): 111-8.

3.- Roberts J A, Sockett P N. The socio-economic impact of human Salmonella enteritidis infection. Int J Food Microbiol 1994; 21 (1-2): 117-29.

4.- Jones K E, Patel N G, Levy M A, Storeygard A, Balk D, Gittleman J L, et al. Global trends in emerging infectious diseases. Nature 2008; 451 (7181): 990-3.

5.- Käferstein F, Abdussalam M. Food safety in the 21st century. Bull World Health Org 1999; 77 (4): 347-51.

6.- Kass P H, Rieman H P. Epidemiology of foodborne diseases. In: H. P. Rieman D. O. Cliver (Eds.). Foodborne infections and intoxications, San Diego: Academic Press 2006; 3-26.

7.- Majowicz S E, Horrocks J, Bocking K. Demographic determinants of acute gastrointestinal illness in Canada: A population study. BMC Public Health 2007; 7: 162.

8.- Mead P S, Slutsker L, Dietz V, McCaig LF, Bresee J S, Shapiro C, et al. Food related illness and death in the United States. Emerg Infect Dis 1999; 5 (5): 607-25.

9.- Centers for Disease Control and Prevention (CDC). Surveillance for foodborne disease outbreaks-United States, 2007. MMWR Morb Mortal Wkly Rep 2010; 59 (31): 973-9.
10.- Scallan E, Majowicz S E, Hall G, Banerjee A, Bowman C L, Daly L, et al. Prevalence of diarrhoea in the community in Australia, Canada, Ireland, and the United States. Int J Epidemiol 2005; 34 (2): 454-60.

11.- Scallan E. Activities, achievements, and lessons learned during the first 10 years of the Foodborne Diseases Active Survillance Network: 1996-2005. Clin Infect Dis 2007; 44 (5): 718-25.

12.- Thomas M K, Pérez E, Majowicz S E, Reid-Smith R, Olea A, Díaz J, et al. Burden of acute gastrointestinal illness in the Metropolitan Region, Chile. 2008. Epidemiol Infect 2011; 139 (4): 560-71.

13.- OPS/OMS 1999. Instituto Panamericano de Protección de Alimentos y Zoonosis http:// www.paho.org/spanish/HCP/HCV/doc471.pdf. (Consultado: 19/04/2011).

14.- Ministerio de Salud de Chile. Reglamento sobre Notificación de Enfermedades Transmisibles de Declaración Obligatoria. Decreto Supremo No 158. Departamento Asesoría Jurídica: Ministerio de Salud. Chile. 22 de octubre de 2004. Disponible en: http:// epi.minsal.cl/epi/html/frames/frame1.htm. (Consultado: 20/04/2011).

15.- Disponible en: http://www.cdc.gov/od/hissb act int.htm. (Consultado: 20/04/2011). CDC. Health Information and Surveillance System Board. Disease Surveillance System Emerg Infect Dis 2006.

16.- OPS. Vigilancia Epidemiológica. En: Principios de Epidemiología para el Control de Enfermedades. Unidad de Epidemiología. Programa ampliado de libros de texto de la OMS. 2000.

17.- Buehler J W, Hopkins R S, Overhage J M, Sosin D M, Tong V; CDC Working Group. Framework for evaluating public health surveillance systems for early detection of outbreaks: recommendations from the CDC
Working Group. MMWR Recomm Rep 2004; 53 (RR-5): 1-11.

18.- Helms M, Simonsen J, Mølbak K. Foodborne bacterial infection and hospitalization: a registry-based study. Clin Infect Dis 2006; 42 (4): 498-506.

19.- Centers for Disease Control and Prevention (CDC). Preliminary FoodNet data on the incidence of infection with pathogens transmitted commonly through food-10 states, 2006. MMWR Morb Mortal Wkly Rep 2007; 56 (14): 336-9.

20.- Díaz J, Solari V, Cáceres O, Mena J, Baeza S, Muñoz X, et al. Brote de gastroenteritis aguda en la región de Antofagasta, Chile, 2010. Rev Chilena Infectol 2012; 29: 19-25.

21.- Instituto Nacional de Estadísticas. Encuesta de calidad de vida de los chilenos. MINSAL. 2008. Disponible:http:/www.ine.cl/canales/ sala_prensa/noticias/2006/marzo/not160306. php. (Consultado: 14/02/2011).

22.- Centers for Disease Control and Prevention (CDC). Preliminary FoodNet data on the incidence of infection with pathogens transmitted commonly through food-10 states, 2009. MMWR Morb Mortal Wkly Rep 2010; 59 (14): 418-22.

23.- Código de prácticas de higiene para los huevos y los productos de huevo. Codex Alimentarius FAO, CAC/RCP 15-1976. Revisión 2007; 5-6.

24.- Centers for Disease Control and Prevention (CDC). Preliminary FoodNet data on the incidence of infection with pathogens transmitted commonly through food - 10 states, 2007. MMWR Morb Mortal Wkly Rep 2008; 57 (14): 366-70.

25.- Olea A. Las enfermedades transmitidas por alimentos: un fenómeno frecuente de magnitud real desconocida. Boletín Vigilancia Epidemiológica MINSAL. El Vigía 25. 2006; 37-42. 